\title{
Deformations of noncompact Calabi-Yau manifolds, families and diamonds
}

\author{
Elizabeth Gasparim and Francisco Rubilar
}

\begin{abstract}
We discuss a new notion of deformation of complex structure, which we use as an adaptation of Kodaira's theory of deformations, but that is better suited to the study of noncompact manifolds. We present several families of deformations illustrating this new approach. Our examples include toric Calabi-Yau threefolds, cotangent bundles of flag manifolds, and semisimple adjoint orbits. We describe their Hodge theoretical invariants, depicting Hodge diamonds and KKP diamonds.
\end{abstract}

\section{Deformations of complex structures}

We present a survey of recent results on deformations of noncompact complex manifolds giving a variety of examples. We explore some new Hodge theoretical invariants of Landau-Ginzburg models, motivated by the KKP conjecture. This conjecture states that three invariants arising from a priori unrelated fundamental ideas in complex geometry actually coincide. It has been recently proved that this conjecture is false in full generality, but as shown in the examples in this paper, there are important families where it does hold. Some families of deformations treated in this survey come from adjoint orbits of certain complex semisimple Lie algebras. We focus on the noncompact case, which turns out to behave radically different from the compact one.

Deformation theory of complex structures for compact manifolds was initially developed by K. Kodaira and D.C. Spencer in the second half of the twentieth century. Their seminal work is summarized in the textbook "Complex manifolds and deformations of complex structures" [Ko]. We recall some of the main results of the compact case, explain some difficulties encountered when using the same definition for the noncompact case, and then state the definition we propose Def. 2.3, which seems better suited to the case of noncompact manifolds. We give several examples initially in two and three complex dimensions, and then in further generality using adjoint orbits and cotangent bundles, we give examples in any complex dimension.

2010 Mathematics Subject Classification. 32G05.

E. Gasparim was partially supported by Simons Associateship grant of the Abdus Salam International Centre for Theoretical Physics and Network grant NT8 of the Office of External Activities, ICTP.

F. Rubilar was partially supported by Beca Doctorado Nacional Conicyt Folio 21170589 and Network grant NT8 of the Office of External Activities, ICTP. 
1.1. The compact case. Let us recall some fundamental results from the theory of classical deformations of complex structures for compact manifolds. The intuitive idea underlying the deformation theory of complex manifolds goes as follows. As Kodaira states in [Ko, Sec. 4.1], roughly speaking, a finite dimensional complex manifold $M=M^{n}$ is obtained gluing domains $U_{1}, \ldots, U_{j}, \ldots$ in $\mathbb{C}^{n}$, i.e., $M=\bigcup_{j} U_{j}$. By $[\mathbf{K o}$, Thm. 2.1], these domains can be considered as polydisks. Hence, if $M$ is a compact complex manifold and $\mathfrak{U}=\left\{U_{j}\right\}$ is an open covering of $M$, then we can assume $\mathfrak{U}$ finite. Thus a compact complex manifold $M$ is obtained by glueing a finite number of polydisks $U_{1}, \ldots, U_{j}, \ldots, U_{l}$ by identifying $z_{k} \in U_{k}$ and $z_{j}=f_{j k}\left(z_{k}\right) \in U_{j}: M=\bigcup_{j=1}^{l} U_{j}$ where $f_{j k}$ denotes the transition function associated to $U_{j}, U_{k} \in \mathfrak{U}$ such that the intersection $U_{j} \cap U_{k}$ is not empty. Hence, roughly speaking a deformation of $M$ is considered to be the glueing of the same polydisks $U_{j}$ via different identifications, i.e., replacing $f_{j k}\left(z_{k}\right)$ by $f_{j k}\left(z_{k}, t\right)$ where $t$ is called the parameter of deformation. Determining when and how the structure of $M$ as a complex manifold depends on this parameter $t$ introduced in the transition functions is the fundamental idea behind the concept of deformations of complex structures.

Now we recall two basic definitions in deformation theory, namely the concepts of family and of infinitesimal deformation.

Definition 1.1. [Ko, Def. 2.8] Suppose given a compact complex manifold $M_{t}=$ $M_{t}^{n}$ for each point $t$ of a domain $B$ of $\mathbb{C}^{m}$. A smooth manifold $\mathcal{M}=\left\{M_{t} \mid t \in B\right\}$ is called an analytic family of compact complex manifolds if there exists a holomorphic surjection $\bar{\omega}: \mathcal{M} \rightarrow B$ satisfying the following conditions:

$\iota$. For each $t \in B, \bar{\omega}^{-1}(t)$ is a compact connected subset of $\mathcal{M}$.

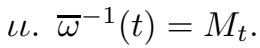

$\iota \iota \iota$. The rank of the Jacobian matrix of $\bar{\omega}$ equals $m$ at every point of $\mathcal{M}$.

$\iota \nu$. There are a locally finite open covering $\left\{U_{j} \mid j=1,2, \ldots\right\}$ of $\mathcal{M}$ and complex-valued holomorphic functions $z_{j}^{1}(p), \ldots, z_{j}^{n}(p), j=1,2, \ldots$, defined on $U_{j}$ such that for each $t$

$$
\left\{p \rightarrow z_{j}^{1}(p), \ldots, z_{j}^{n}(p) \mid U_{j} \cap \bar{\omega}^{-1}(t) \neq \emptyset\right\}
$$

forms a system of local complex coordinates of $M_{t}$.

By $\iota$. and $\iota$. each $\bar{\omega}^{-1}(t)$ is a compact differentiable manifold. Condition $\iota \iota \iota$. means that $\bar{\omega}^{-1}(t)$ is the underlying differentiable manifold of $M_{t}$. With this notation $t \in B$ is the parameter of the analytic family $\left\{M_{t} \mid t \in B\right\}$ and $B$ its parameter space or base space. This definition can be extended to the case when $B$ is an arbitrary complex manifold.

Notation 1.2. With the same notation as before, in order to make explicit the parameter space it is usual to write down an analytic complex family as $(\mathcal{M}, \bar{\omega}, B)$.

Definition 1.3. [Ko, Def. 2.9] Let $M$ and $N$ be two compact complex manifolds. $N$ is called a deformation of $M$ if $M$ and $N$ belong to the same complex analytic family, that is, if there is a complex analytic family $(\mathcal{M}, \bar{\omega}, B)$ with a complex manifold $B$ as its parameter space such that $M=\bar{\omega}^{-1}\left(t_{0}\right)$ and $N=\bar{\omega}^{-1}\left(t_{1}\right)$ for some $t_{0}, t_{1} \in B$.

With the above definition in mind we get that any two elements of an analytic complex family are diffeomorphic [Ko, Thm. 2.3]. Hence, it follows that the 
differentiable structure of complex manifolds does not change under deformation. Several examples of deformations can be found in [Ko, Sec. 2.3].

The fundamental issue when we consider deformations of complex manifolds is the following: given an analytic family $(\mathcal{M}, \bar{\omega}, B)$ of compact complex manifolds, how can we determine whether the complex structure of $M_{t}=\bar{\omega}^{-1}$ actually depends on $t$ ? Essentially the approach goes via differentiating the transition functions with respect to the parameter $t$. Accordingly, it is possible to show that deformations of a compact complex manifold $M$ are parametrized by $\mathrm{H}^{1}(M, T M)$, that is, the first cohomology group with coefficient in its tangent sheaf. Thus, if $\theta$ is any nonzero class in $\mathrm{H}^{1}(M, T M)$, we can associate to $\theta$ a deformation of $M$ which is called an infinitesimal deformation. In general, computing the dimension of the first cohomology group with coefficients in the tangent sheaf is not enough to prove existence of deformation of a compact complex manifold. It is also necessary verify integrability, that is, if there exist manifolds realizing such directions of deformations, and it is also necessary to check whether there are obstructions which correspond to elements in $\mathrm{H}^{2}(M, T M)$, [Ko, Thm. 5]. We exhibit how this works in some examples.

Example 1.4. The complex projective space $\mathbb{P}^{n}$ has no (classical) deformations of its complex structure. This result follows directly from the fact that $\mathrm{H}^{1}\left(\mathbb{P}^{n}, T \mathbb{P}^{n}\right)=$ 0 and $\mathbb{P}^{n}$ is compact. For instance, if we consider $n=1$, we have that $T \mathbb{P}^{1} \cong \mathcal{O}_{\mathbb{P}^{1}}(2)$. Since $\mathrm{H}^{1}\left(\mathbb{P}, \mathcal{O}_{\mathbb{P}^{1}}(2)\right)=0$, the result follows.

Example 1.5. If $M$ is a curve of genus $g$, then $\mathrm{H}^{2}(M, T M)=0$. Hence there are no obstructions to deforming its complex structure.

Example 1.6. The Hirzebruch surface $\mathbb{F}_{k}$ defined as

$$
\mathbb{F}_{k}=\operatorname{Proj}\left(\mathcal{O}_{\mathbb{P}^{1}}(-k) \oplus \mathcal{O}_{\mathbb{P}^{1}}\right), \quad k \geq 0
$$

is compact and has $\left\lfloor\frac{k}{2}\right\rfloor$ deformations, these are the $\mathbb{F}_{k-2 i}$.

\section{Deformations of noncompact manifolds}

In this section we describe our methods of finding infinitesimal deformations of noncompact manifolds which were used in $[\mathbf{B G 1 , ~ R}]$ and $[$ GKRS]. When looking for deformations of noncompact manifolds one needs to keep in mind the caveat that cohomology calculations are generally not enough to decide questions of existence of infinitesimal deformations, as the following example illustrates. Suppose we naively extended Definition 1.1 to the noncompact case by simply removing the requirements of compactness, that is, repeating the same definition only with all appearances of the word compact deleted. This would work poorly, as the following example shows.

Example 2.1. Edoardo Ballico gave us the following illustration that cohomological rigidity may not imply absence of deformations,

We consider deformations of $X=\mathbb{C}$. Clearly $\mathrm{H}^{1}(X, T X)=0$. However, with the naive generalizations form the compact case, there do exist nontrivial deformations of $X$ as the following family shows.

Consider $\pi: \mathbb{P}^{1} \times D \rightarrow D$ with $D$ any smooth manifold (even $\mathbb{P}^{1}$ or a disc) and a specific $o \in D$. Take $s_{\infty}: D \rightarrow \mathbb{P}^{1} \times D$ the section of $\pi$ defined by

$$
s_{\infty}(x)=(\infty, x)
$$


then take another section $s$ of $\pi$ with:

$$
s(o)=(\infty, o), \quad s(x)=\left(a_{x}, x\right)
$$

with $a_{x} \in \mathbb{C}=\mathbb{P}^{1} \backslash\{\infty\}$. Take as the total space for our family $Y$ as $\mathbb{P}^{1} \times D$ minus the images of the two sections. Then we obtain a deformation of $\mathbb{C}$ in which at all points of $D \backslash\{o\}$ you have $\mathbb{C} \backslash\{0\}$. Thus not a trivial deformation in any reasonable sense.

Remark 2.2. Example 2.1 shows that vanishing of cohomology may not imply nonexistence of deformations, at least not if we allow the smooth type of the manifold to vary in the family. Nevertheless, as we shall see, cohomology calculations are still useful to find deformations.

In this work by deformation we mean the following:

Definition 2.3. A deformation family of a complex manifold $X$ is a holomorphic surjective submersion $\tilde{X} \stackrel{\pi}{\rightarrow} D$, where $D$ is a complex disc centred at 0 (possibly a vector space, possibly infinite dimensional), satisfying:

- $\pi^{-1}(0)=X$,

- $\tilde{X}$ is locally trivial in the $C^{\infty}$ category.

The fibres $X_{t}=\pi^{-1}(t)$ are called deformations of $X$.

In further generality, allowing the parameter space to be a variety or a scheme, and requiring that the bundle be locally trivial, we obtain the concept of a family of complex structures.

Remark 2.4. We say that a deformation is trivial if it is isomorphic in the holomorphic category to the original manifold.

Remark 2.5. Our choice for the dimension of $D$ is $n=h^{1}(X, T X)$ whenever possible. The case $n=0$ may be included in the following definition.

Definition 2.6. We call a manifold $X$ formally rigid when $\mathrm{H}^{1}(X, T X)=0$.

Definition 2.7. We call a manifold $X$ rigid if any deformation $\tilde{X} \stackrel{\pi}{\rightarrow} D$ is biholomorphic to the trivial bundle $X \times D \rightarrow D$.

Example 2.8. Projective spaces are rigid. Indeed, Ex. 1.4 shows it is formally rigid, and in the compact case formally rigid implies rigid.

In general, formally rigid does not imply rigid, as example 2.1 shows, but we improve on the situation with our proposed definition. With Def. 2.3 we do not claim to solve the problem that a manifold $X$ does not deform under the condition $\mathrm{H}^{1}(X, T X)=0$, however we certainly eliminate some unwanted pathological cases such as the one in Example 2.1.

Observe that the deformations considered in [BG1] satisfy Definition 2.3, hence maintain the $C^{\infty}$ type of the manifold.

2.1. Surfaces. We give examples of noncompact surfaces which have finite dimensional spaces of deformations, and exhibit their families of deformations. Let $Z_{k}$ be the noncompact surface defined as

$$
Z_{k}=\operatorname{Tot}\left(\mathcal{O}_{\mathbb{P}^{1}}(-k)\right) ; \quad k \geq 1 .
$$

Barmeier and Gasparim [BG1] described the classical deformation theory of the surfaces $Z_{k}$, they have also described their noncommutative deformation theory in 
[BG2], but in this work we will restrict ourselves to classical deformations. Here we recall a definition taken from $[\mathbf{M}]$, in order to clarify the exposition of the results for the non-compact surfaces $Z_{k}$.

These surfaces are total spaces of negative line bundles on the projective line. A preliminary estimate via cohomology calculations [BG1, Thm. 5.4] showed that $Z_{k}$ admits a $(k-1)$-dimensional semiuniversal family of classical deformations. Here by semiuniversal it is meant that the family is versal and the Kodaira-Spencer map is bijective, see $[\mathbf{M}]$. Denoting by $\mathcal{Z}_{k}$ any nontrivial deformation of $Z_{k}$ for $k \geq 2$, [BG1, Thm.6.6] showed that $\mathcal{Z}_{k}$ contains no compact complex analytic curves. Furthermore, [BG1, Thm. 6.14] showed that any holomorphic vector bundle on $\mathcal{Z}_{k}$ splits as a direct sum of algebraic line bundles. This is somewhat surprising, given the existence of nontrivial moduli of vector bundles on the original $Z_{k}$ surfaces as constructed in $[\mathbf{B G K}]$. Also in $[\mathbf{B G 1}]$ it is proved that any nontrivial deformation of $Z_{k}$ is affine (this situation contrasts with the case of the threefolds considered in the next section).

Deformation theory effects on moduli of vector bundles have applications to mathematical physics. The effects for the case of surfaces $Z_{k}$ can be interpreted in Yang-Mills theory. They imply that moduli of $S U(2)$ instantons on noncompact surfaces are sensitive to the complex structure: the moduli of irreducible instantons of normalized charge (or splitting) $j$ over the noncompact surfaces $Z_{k}$ are of dimension $2 j-k-2$, whereas a nontrivial (commutative) deformation of $Z_{k}$ admits no instantons [BG1, Thm. 7.4]. In comparison, in the context of deformations of compact curves and surfaces and their moduli we note that by Grothendieck's splitting theorem any holomorphic vector bundle on $\mathbb{P}^{1}$ splits as a direct sum of (algebraic) line bundles. Neither the curve $\mathbb{P}^{1}$ itself nor its moduli spaces of vector bundles admit any deformations. Curves of higher genus do admit deformations and a celebrated theorem of Narasimhan and Ramanan $[\mathbf{N R}, \mathbf{B V}]$ shows that all classical deformations of the moduli of stable bundles on a smooth curve come from deformations of the curve itself (case $g>1, \quad(r, d)=1$ ). As we saw, deformations of the surfaces $Z_{k}$ does not produce new moduli of bundles. The situation for Calabi-Yau threefolds turns out a lot more interesting, as we shall now see.

2.2. Calabi-Yau threefolds. We define Calabi-Yau threefolds $W_{k}$ as total spaces of holomorphic vector bundles on $\mathbb{P}^{1}$, i.e.,

$$
W_{k}=\operatorname{Tot}(\mathcal{O}(-k) \oplus \mathcal{O}(k-2)) ; \quad k \geq 1 .
$$

We put canonical coordinates on $W_{k}$ by taking the open covering $\mathfrak{U}=\{U, V\}$, where $U=\left\{\left(z, u_{1}, u_{2}\right)\right\} \simeq \mathbb{C}^{3} \simeq V=\left\{\left(\xi, v_{1}, v_{2}\right)\right\}$ such that

$$
\left(\xi, u_{1}, u_{2}\right)=\left(z, z^{k} u_{1}, z^{2-k} u_{2}\right),
$$

on the intersection $U \cap V \simeq \mathbb{C}^{*} \times \mathbb{C}^{2}$. The behaviour of the deformations of these manifolds is quite different from the one of deformations of the surfaces $Z_{k}$. This situation is described by the following results proved by Rubilar in $[\mathbf{R}]$ and Gasparim and Suzuki in $[\mathbf{G S}]$. First, Rubilar proved that while $W_{1}$ is formally rigid $[\mathbf{R}$, Lem. 5.2.1], $W_{2}$ has an infinite dimensional family of deformations [R, Thm. 6.3.2]. The latter was accomplished by showing that $\operatorname{dim} \mathrm{H}^{1}\left(W_{2}, T W_{2}\right)=\infty$ and then proving that directions of deformations parametrized by such cohomology are integrable, by explicitly constructing the corresponding families. Moreover, he also 
showed that $W_{2}$ has both affine and non-affine deformations, situation which contrasts with the one of the surfaces $Z_{k}$. Subsequently, Gasparim and Suzuki showed explicitly that there are infinitely many isomorphism types among the deformations of $W_{2}$ [GS, Thm. 3.7]. Then, presenting transformations taking deformations of $W_{k}$ for $k>2$ to deformations of $W_{2}$ and conversely, they obtain also infinitely many isomorphism types of deformations of $W_{k}$ for $k>2$.

2.2.1. Moduli of vector bundles. The study of deformations is very closely linked to the theory of moduli spaces. In fact, there is a precise sense in which infinitesimal deformations can be used to generate neighborhoods of moduli spaces. For the case of vector bundles this is known as Kuranishi theory. Here we only comment on the results for our surfaces and threefolds, without discussing the general theory.

For deformations of surfaces $Z_{k}$, Barmeier and Gasparim showed that moduli spaces of holomorphic vector bundles with fixed topological invariants are trivial [BG1, Cor. 6.19], i.e. consist of a single point. For the threefold $W_{2}$, Gasparim and Suzuki showed that some nontrivial deformations of $W_{2}$ have the effect of decreasing the dimension of the moduli of vector bundles by just one dimension, hence keeping nontrivial moduli [GS, Thm. 8.13].

In conclusion, we have observed that the theories of deformations of surfaces $Z_{k}$ and threefolds $W_{k}$ are qualitatively different, the latter being very rich in terms of applications to moduli of vector bundles.

\section{Hyperkähler families}

Definition 3.1. A hyperkähler manifold is a Riemannian manifold of real dimension $4 k$ and holonomy group contained in $\operatorname{Sp}(k)$.

Here $\operatorname{Sp}(k)$ denotes a compact form of a symplectic group, identified with the group of quaternionic-linear unitary endomorphisms of a $k$-dimensional quaternionic Hermitian space. Every hyperkähler manifold $M$ has a 2-sphere of complex structures with respect to which the metric is Kähler.

In particular, it is a hypercomplex manifold, meaning that there are three distinct complex structures, $I, J$, and $K$, which satisfy the quaternion relations

$$
I^{2}=J^{2}=K^{2}=I J K=-1 .
$$

Any linear combination

$$
a I+b J+c K
$$

with $a, b, c$ real numbers such that $a^{2}+b^{2}+c^{2}=1$ is also a complex structure on $M$. Hence, hyperkähler manifolds are especially rich examples where to study deformations of complex structures. Each of the complex structures $a I+b J+c K$ may be regarded as a deformation of $I$.

Remark 3.2. To start with $I, J, K$ are almost complex structures, these are then required to be integral, and in such case the complex structures obtained agree with the ones defined by Kodaira using charts.

Remark 3.3. We have the following general facts:

(1) Hyperkähler manifolds are special classes of Kähler manifolds.

(2) All hyperkähler manifolds are Ricci-flat and are thus Calabi-Yau manifolds. 
Compact hyperkähler manifolds have been extensively studied using techniques from algebraic geometry, sometimes under the alternative name of holomorphically symplectic manifolds. Due to Fedor Bogomolov's decomposition theorem $(1974,[\mathbf{B o}])$, the holonomy group of a compact holomorphically symplectic manifold $M$ is exactly $\operatorname{Sp}(k)$ if and only if $M$ is simply connected and any pair of holomorphic symplectic forms on $M$ are scalar multiples of each other.

For applications to the study of Hodge theory of Landau-Ginzburg (LG) models (see Def. 4.1), here we wish to focus on those hyperkähler families that contain the adjoint orbits of semisimple Lie groups. An adjoint orbit of a compact Lie group is called a flag manifold.

Families of hyperkähler structures containing semisimple adjoint orbits were studied by Kronheimer $[\mathbf{K r} \mathbf{1}]$, Biquard $[\mathbf{B q}]$, and Kovalev $[\mathbf{K v}]$. Such families include both cotangent bundles of flag manifolds and adjoint orbits of semisimple Lie algebras. In the following section, we will add superpotentials to these families and then study them from the point of view of Landau-Ginzburg models. The approach we propose here goes in a different way, we study the hyperkählerian structures using Lie theory techniques. We first recall some definitions and results.

TheOREM 3.4. [Kv, Thm. 1.1] Let $G$ be a compact semisimple Lie group with $\mathfrak{g}$ its Lie algebra, $H$ a stabilizer of some non-zero element of $\mathfrak{g}$ under the adjoint action of $G$, and $T$ a maximal torus such that $G \supseteq H \supseteq T$. Let $G^{c}, H^{c}$ be the complex forms of $G$ and $H$. Then the manifold $G^{c} / H^{c}$ has a family of hyperkählerian structures with the following properties:

(1) (a) the hyperkählerian metrics are complete,

(b) the family admits parametrisation as follows: let $\mathfrak{t}$ be a Cartan subalgebra of $\mathfrak{g}$ corresponding to $T$, then the parameter space consists of those triples $\left(\tau_{1}, \tau_{2}, \tau_{3}\right)$ in $\mathfrak{t}$ which each have the stabilizer $H$;

(2) On $G^{c} / H^{c}$ there is a complex structure I which is independent of $\tau_{1}$. If also the complex group $H^{c}$ is the stabilizer of the element $\tau^{c}=\tau_{2}+i \tau_{3}$ then the underlying complex manifold determined by $I$ is isomorphic to the complex adjoint orbit $\operatorname{Ad}\left(G^{c}\right) \tau^{c}$.

Remark 3.5. Observe that putting $H^{c}=T^{c}$ we obtain the result of Kronheimer [Kr1] on regular semisimples orbits, that is, those of type $G^{c} / T^{c}$. In general $T^{c}$ is a proper subgroup of $H^{c}$, therefore in such case the family of hyper-Kählerian structures is smaller.

To motivate the parametrisation, consider the adjoint action of $G^{c}$. Let $\tau^{c}$ be some element of $\mathfrak{g}^{c}$ and $H^{c}$ its stabilizer. The orbit $\operatorname{Ad}\left(G^{c}\right) \tau^{c}$ is diffeomorphic to $G^{c} / H^{c}$. The second de Rham cohomology of $G^{c} / H^{c}$ can be identified with a real commutative Lie algebra $\mathfrak{h}_{\tau} \subset \mathfrak{g}$ determined (up to isomorphism) by regularity properties of $\tau^{c}$ : for example, $\mathfrak{h}_{\tau}=\mathfrak{t}$ if $\tau^{c}$ is a regular element of $\mathfrak{t}^{c}$. The three Kähler structures on a complex orbit and the cohomology classes of their Kähler forms can be put in correspondence to elements $\tau_{1}, \tau_{2}, \tau_{3}$ in $\mathfrak{h}_{\tau}$ such that $\tau_{2}+i \tau_{3}=\tau^{c}$. Then $\tau_{1}$ serves as a parameter in the family of hyperkählerian structures induced on $G^{c} / H^{c}$ from the orbit of $\tau_{2}+i \tau_{3}$.

Theorem 3.6. [Kv, Thm.1.2] Let $G$ and $\mathfrak{g}$ be as in Thm. 3.4. If $\tau_{2}, \tau_{3} \in \mathfrak{t}$ and $\sigma^{c}$ is a nilpotent element of the centralizer $z\left(\tau_{2}+i \tau_{3}\right)$ in $\mathfrak{g}^{c}$ then the complex adjoint orbit $\operatorname{Ad}\left(G^{c}\right)\left(\tau_{2}+i \tau_{3}+\sigma^{c}\right)$ has a family of hyperkählerian structures. The 
family is parametrised by elements $\tau_{1} \in \mathfrak{t}$ such that the centralizers in $\mathfrak{g}$ of the pair $\left(\tau_{2}, \tau_{3}\right)$ and the triple $\left(\tau_{1}, \tau_{2}, \tau_{3}\right)$ coincide.

Remark 3.7. If $\tau_{2}=\tau_{3}=0$ then $\tau_{1}=0$ is the only possible value and we obtain the following result due to Kronheimer.

Corollary 3.8. [Kr2] A nilpotent adjoint orbit of $G^{c}$ is a hyperkähler manifold.

It remains to point out that Theorem 3.6 applies to all adjoint orbits of $G^{c}$. This follows immediately from standard properties of complex semisimple Lie algebras, the details can be found e.g. in Humphreys $[\mathbf{H}]$ or San Martin $[\mathbf{S M}]$. Any element of $\mathfrak{g}^{c}$ can be written uniquely in the form $x=s+n$, with $n$ nilpotent, $s$ semisimple and $[s, n]=0$. As a semisimple element $s$ is contained in some Cartan subalgebra, that is in $\operatorname{Ad}(g) \mathfrak{t}^{c}$ for some $g \in G^{c}$. So any element of $\mathfrak{g}^{c}$ is $G^{c}$-conjugate to one of the form $\left(\tau_{2}+i \tau_{3}\right)+\sigma^{c}$.

We state in a simplified form of a consequence of Thm. 3.4 which we will use to study deformations.

Corollary 3.9. Let $\mathfrak{g}$ be a semisimple complex Lie algebra, and $H_{o} \in \mathfrak{g}$ a regular element. Let $G$ be a connected Lie group with Lie algebra $\mathfrak{g}$ and $K$ a compact form of $G$. Then the adjoint orbit $\operatorname{Ad} G\left(H_{0}\right)$ is a deformation of the cotangent bundle of the flag manifold Ad $K\left(H_{0}\right)$.

\section{Adjoint orbits and cotangent bundles}

Using the result of Thm. 3.4 we see that adjoint orbits may be regarded as deformations of cotangent bundles of flag manifolds. That is, if $G$ is a complex Lie group and $K$ its compact part, then an adjoint orbit $\operatorname{Ad} G\left(H_{o}\right)$ can be regarded as a deformation of the cotangent bundle of the flag manifold $\mathrm{Ad} K\left(H_{0}\right)$. We wish to add superpotentials to these families, to look at them from the point of view of Landau-Ginzburg models, and then to study how their Hodge diamonds vary in the families. We first recall the construction given in [GGSM1].

Definition 4.1. A Landau-Ginzburg model (LG) is a pair $(Y, w)$, where

(1) $Y$ is a smooth complex quasi-projective variety with trivial canonical bundle $K_{Y}$;

(2) $w: Y \rightarrow \mathbb{C}$ is a morphism with a compact critical locus $\operatorname{crit}(w) \subset Y$, called the superpotential.

4.1. LG models on adjoint orbits. Let $\mathfrak{g}$ be a complex semisimple Lie algebra with Cartan subalgebra $\mathfrak{h}$, and $\mathfrak{h}_{\mathbb{R}}$ the real subspace generated by the roots of $\mathfrak{h}$. Let $\Pi$ denote the set of all roots of $\mathfrak{h}$. An element $H \in \mathfrak{h}$ is called regular if $\alpha(H) \neq 0$ for all $\alpha \in \Pi$.

Notation 4.2. $\mathcal{O}\left(H_{0}\right):=\operatorname{Ad} G\left(H_{o}\right)$.

Theorem 4.3. [GGSM1, Thm.3.1] Given $H_{0} \in \mathfrak{h}$ and $H \in \mathfrak{h}_{\mathbb{R}}$ with $H$ a regular element, the potential $f_{H}: \mathcal{O}\left(H_{0}\right) \rightarrow \mathbb{C}$ defined by

$$
f_{H}(x)=\langle H, x\rangle \quad x \in \mathcal{O}\left(H_{0}\right)
$$

has a finite number of isolated singularities and defines a symplectic Lefschetz $f$ bration; that is to say

(1) the singularities are (Hessian) nondegenerate; 
(2) if $c_{1}, c_{2} \in \mathbb{C}$ are regular values then the level manifolds $f_{H}^{-1}\left(c_{1}\right)$ and $f_{H}^{-1}\left(c_{2}\right)$ are diffeomorphic;

(3) there exists a symplectic form $\Omega$ on $\mathcal{O}\left(H_{0}\right)$ such that the regular fibres are symplectic submanifolds;

(4) each critical fibre can be written as the disjoint union of affine subspaces contained in $\mathcal{O}\left(H_{0}\right)$, each symplectic with respect to $\Omega$.

Given the Iwasawa decomposition: $\mathfrak{g}=\mathfrak{k} \oplus \mathfrak{a} \oplus \mathfrak{n}$, if $\Pi$ is a set of roots of $\mathfrak{a}$, with a choice of a set of positive roots $\Pi^{+}$and simple roots $\Sigma \in \Pi^{+}$, then the corresponding Weyl chamber is $\mathfrak{a}^{+}$. A subset $\Theta \in \Sigma$ defines a parabolic subalgebra $\mathfrak{p}_{\Theta}$ with parabolic subgroup $P_{\Theta}$ and a flag $\mathbb{F}_{\Theta}=G / P_{\Theta}$. A element $H_{\Theta} \in c l \mathfrak{a}^{+}$is characteristic for $\Theta \subset \Sigma$ if $\Theta=\left\{\alpha \in \Sigma: \alpha\left(H_{\Theta}\right)=0\right\}$.

ThEOREM 4.4. [GGSM2, Thm. 2.1] The adjoint orbit $\mathcal{O}\left(H_{\Theta}\right)=\operatorname{Ad}(G) \cdot H_{\Theta} \simeq$ $G / Z_{\Theta}$ of the characteristic element $H_{\Theta}$ is a $C^{\infty}$ vector bundle over $\mathbb{F}_{\Theta}$ isomorphic as a vector bundle to the cotangent bundle $T^{*} \mathbb{F}_{\Theta}$. Moreover, we can write down a diffeomorphism $\iota: \operatorname{Ad}(G) \cdot H_{\Theta} \rightarrow T^{*} \mathbb{F}_{\Theta}$ such that

(1) ८ is equivariant with respect to the actions of $K$, that is, for all $k \in K$,

$$
\iota \circ \operatorname{Ad}(k)=\widetilde{k} \circ \iota
$$

where $K$ is the compact subgroup in the Iwasawa decomposition $G=$ $K A N$, and $\widetilde{k}$ is the lifting to $T^{*} \mathbb{F}_{\Theta}$ (via the differential) of the action of $k$ on $\mathbb{F}_{\Theta}$.

(2) The pullback of the canonical symplectic form on $T^{*} \mathbb{F}_{\Theta}$ by $\iota$ is the (real) Kirillov-Kostant-Souriau form on the orbit.

Viewing the orbit as the cotangent bundle of a flag manifold, we can identify the topology of the fibres in terms of the topology of the flag. Denote by $\mathcal{W}$ the Weyl group of $G$, then we have the following two corollaries.

Corollary 4.5. [GGSM1, Cor.4.5] The homology of a regular fibre coincides with the homology of $\mathbb{F}_{\Theta} \backslash \mathcal{W} \cdot H_{\Theta}$. In particular the middle Betti number is $k-1$ where $k$ is the number of singularities of the fibration (equal to the number of elements in $\left.\mathcal{W} \cdot H_{\Theta}\right)$.

For the case where singular fibres have only one critical point, we have the following corollary.

Corollary 4.6. [GGSM1, Cor.5.1] The homology of the singular fibre though $w H_{\Theta}, w \in \mathcal{W}$, coincides with that of

$$
\mathbb{F}_{H_{\Theta}} \backslash\left\{u H_{\Theta} \in \mathcal{W} \cdot H_{\Theta} \mid u \neq w\right\} .
$$

In particular, the middle Betti number of this singular fibre equals $k-2$, where $k$ is the number of singularities of the fibration $f_{H}$.

These corollaries show that Hodge diamonds for the LG models can have arbitrarily high numbers in their middle cohomology, and that these Lefschetz fibrations may have large quantities of vanishing cycles. 


\section{Diamonds}

5.1. Hodge diamonds. We now wish to consider some examples of how diamonds vary under deformations. Consider first the standard Hodge diamond of a variety:

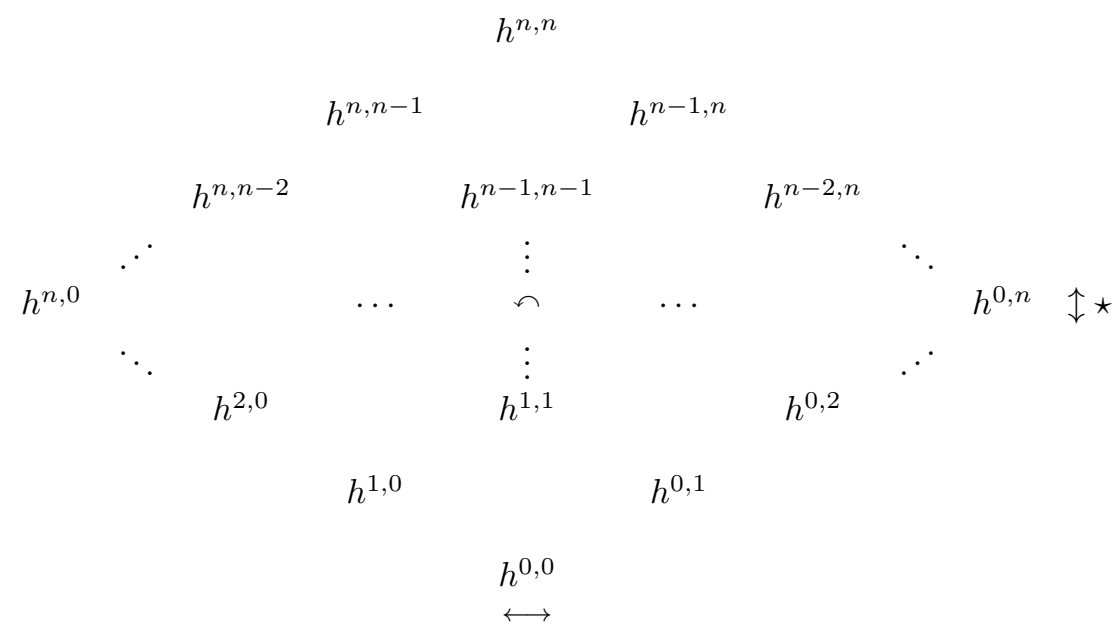

where the symbols $\curvearrowleft, \longleftrightarrow, \uparrow \star$ are there to remind us that in the case of smooth projective varieties there are symmetries of the diamond corresponding to Serre duality, conjugation, and Hodge star, respectively.

5.2. KKP diamonds. For the case of Landau-Ginzburg models $(Y, w)$ there are three new Hodge theoretical invariants which were defined by Katzarkov, Kontsevich, and Pantev in $[\mathbf{K K P}]$. These invariants take into account not just the variety, but also the potential together with its critical points and vanishing cycles. They are the numbers $f^{p, q}(Y, w)$ which come from sheaf cohomology of logarithmic forms, the numbers $h^{p, q}(Y, w)$ motivated by mirror symmetry considerations, and the numbers $i^{p, q}(Y, w)$ defined using ordinary mixed Hodge theory.

To define these new invariants, the authors require that the LG model be tamely compactifiable in the following sense.

Definition 5.1. [KKP] A tame compactified Landau-Ginzburg model is the data $\left((Z, f), D_{Z}\right)$, where

(1) $Z$ is a smooth projective variety and $f: Z \rightarrow \mathbb{P}^{1}$ is a flat morphism.

(2) $D_{Z}=\left(\cup_{i} D_{i}^{h}\right) \cup\left(\cup_{j} D_{j}^{v}\right)$ is a reduced normal crossings divisor such that

(i) $D^{v}=\cup_{j} D_{j}^{v}$ is a scheme theoretical pole divisor of $f$, i.e. $f^{-1}(\infty)=$ $D^{v}$. In particular $\operatorname{ord}_{D_{j}^{v}}(f)=-1$ for all $j$;

(ii) each component $D_{i}^{h}$ of $D^{h}=\cup_{i} D_{i}^{h}$ is smooth and horizontal for $f$, i.e. $\left.f\right|_{D_{i}^{h}}$ is a flat morphism;

(iii) The critical locus $\operatorname{crit}(f) \subset Z$ does not intersect $D^{h}$.

(3) $D_{Z}$ is an anticanonical divisor on $Z$.

One says that $\left((Z, f), D_{Z}\right)$ is a compactification of the Landau-Ginzburg model $(Y, w)$ if in addition the following holds:

(4) $Y=Z \backslash D_{Z},\left.f\right|_{Y}=w$. 
Remark 5.2. A caveat about algebraic compactifications should be mentioned here. $[\mathbf{B C G}]$ showed that the choice of compactification may have strong effects on the Hodge diamonds. In fact, they give examples when the topology of the compactified regular fibre for $f_{H}$ changes drastically according to the choice of homogenisation of the ideal cutting out the orbit as an affine variety. For the case of the maximal adjoint orbit of $\mathfrak{s l}(3, \mathbb{C})$, namely the one diffeomorphic to the cotangent bundle of the full flag $F(1,2)$, [BCG] give examples of two algebraic compactifications of such an orbit which produce in one case $h^{1,4}=h^{4,1}=16$ and in the other case $h^{1,4}=h^{4,1}=1$. Such radical difference being produced simply by the choice of homogenisation of the ideal defining the adjoint orbit. Thus, one must be very careful when using compactifications to study the Hodge theory of noncompact varieties. This remark also highlights the importance of the careful definition of a tame compactification.

Assume that we are given a Landau-Ginzburg model $(Y, w)$ with a tame compactification $\left((Z, f), D_{Z}\right)$ as above. Denote by $n=\operatorname{dim} Y=\operatorname{dim} Z$ the (complex) dimension of $Y$ and $Z$. Choose a point $b \in \mathbb{C}$ which is near $\infty$ and such that the fibre $Y_{b}=w^{-1}(b) \subset Y$ is smooth. Let us briefly recall the definitions, for more details see $[\mathbf{L P}]$.

5.2.1. $f^{p, q}(Y, w)$. Let $\Omega_{Z}^{\bullet}\left(\log D_{Z}\right)$ denote the logarithmic de Rham complex. For each $a \geq 0$ define the a sheaf $\Omega_{Z}^{a}\left(\log D_{Z}, f\right)$ of $f$-adapted logarithmic forms as:

$$
\Omega_{Z}^{a}\left(\log D_{Z}, f\right)=\left\{\alpha \in \Omega_{Z}^{a}\left(\log D_{Z}\right) \mid d f \wedge \alpha \in \Omega_{Z}^{a+1}\left(\log D_{Z}\right)\right\} .
$$

Definition 5.3. The Landau-Ginzburg Hodge numbers $f^{p, q}(Y, w)$ are defined as follows:

$$
f^{p, q}(Y, w)=\operatorname{dim} \mathrm{H}^{p}\left(Z, \Omega_{Z}^{q}\left(\log D_{Z}, f\right)\right) .
$$

5.2.2. $h^{p, q}(Y, w)$.

Definition 5.4. [LP, Def. 8] Assume that $(Y, w)$ is a Landau-Ginzburg model of Fano type. Consider the relative cohomology $\mathrm{H}^{\bullet}\left(Y, Y_{b}\right)$ with the nilpotent operator $N$ and the induced canonical filtration $W$. The Landau-Ginzburg numbers $h^{p, q}(Y, w)$ are defined as follows:

$$
\begin{aligned}
& h^{p, n-q}(Y, w)=\operatorname{dim} g r_{2(n-p)}^{W, n-a} \mathrm{H}^{n+p-q}\left(Y, Y_{b}\right) \quad \text { if } a=p-q \geq 0, \\
& h^{p, n-q}(Y, w)=\operatorname{dim} g r_{2(n-q)}^{W, n+a} \mathrm{H}^{n+p-q}\left(Y, Y_{b}\right) \text { if } a=p-q<0
\end{aligned}
$$

where $g r$ is the standard grading of the mixed Hodge structure by the weight filtration.

5.2.3. $i^{p, q}(Y, w)$. For each $\lambda \in \mathbb{C}$ one has the corresponding sheaf $\phi_{w-\lambda} \mathbb{C}_{Y}$ of vanishing cycles for the fibre $Y_{\lambda}$. The sheaf $\phi_{w-\lambda} \mathbb{C}_{Y}$ is supported on the fibre $Y_{\lambda}$ and is equal to zero if $\lambda$ is not a critical value of $w$.

Definition 5.5. Assume that the horizontal divisor $D^{h} \subset Z$ is empty, i.e. assume that the map $w: Y \rightarrow \mathbb{C}$ is proper. Then

(1) the Landau-Ginzburg Hodge numbers $i^{p, q}(Y, w)$ are defined as follows:

$$
i^{p, q}(Y, w)=\sum_{\lambda \in \mathbb{C}} \sum_{k} i^{p, q+k} \mathbb{H}^{p+q-1}\left(Y_{\lambda}, \phi_{w-\lambda} \mathbb{C}_{Y}\right)
$$


(2) In the general case denote by $j: Y \hookrightarrow Z$ the open embedding and define similarly

$$
i^{p, q}(Y, w)=\sum_{\lambda \in \mathbb{C}} \sum_{k} i^{p, q+k} \mathbb{H}^{p+q-1}\left(Y_{\lambda}, \phi_{w-\lambda} \mathbf{R} j_{*} \mathbb{C}_{Y}\right) .
$$

Here $\mathbb{H}$ de notes the hypercohomology of the constructible complexes of the sheaves of vanishing cycles, having higher derived images $\phi_{w-\lambda} \mathbf{R} j_{*} \mathbb{C}_{Y}$.

5.3. The KKP conjecture. The KKP conjecture states that the three invariants coincide, that is,

$$
f^{p, q}(Y, w)=h^{p, q}(Y, w)=i^{p, q}(Y, w) .
$$

For $Y$ a specific rational surface with a map $w: Y \rightarrow \mathbb{C}$ such that the generic fibre is an elliptic curve $[\mathbf{L P}]$ Lunts and Przyjalkowski proved the equality $f^{p, q}(Y, w)=$ $h^{p, q}(Y, w)$ and gave an example where $i^{p, q}(Y, w) \neq h^{p, q}(Y, w)$. Thus, in full generality the conjecture is false. Nevertheless, Cheltsov and Przyjalkowski proved KKP the conjecture for Fano threefolds $[\mathbf{C P}]$ and Ballico, Gasparim, Rubilar, and San Martin proved the KKP conjecture for minimal semisimple adjoint orbits [BGRSM].

Definition 5.6. In the cases when the KKP conjecture holds true, the invariants then define a new diamond, which we call the KKP diamond.

We now give some examples of Hodge diamonds and KKP diamonds computed in [BGRSM].

5.3.1. An example in 2 dimensions. Consider the semisimple adjoint orbit $\mathcal{O}_{2}$ of $\mathfrak{s l}(2, \mathbb{C})$. Hence, $\mathcal{O}_{2}$ can be viewed as the affine hypersurface of $\mathbb{C}^{3}$ cut out by the equation $x^{2}+y z=1$, see [BBGGSM, Sec. 2].

The Hodge diamond of $\mathcal{O}_{2}$ is:

$$
\begin{aligned}
& 0
\end{aligned}
$$

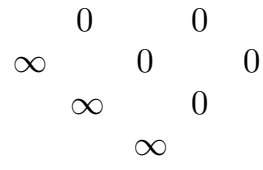

$$
\begin{aligned}
& \mathcal{O}_{2}
\end{aligned}
$$

We know by Cor. 3.9 that $\mathcal{O}_{2}$ is a deformation of $T^{*} \mathbb{P}^{1}$. The Hodge diamond of $\mathrm{T}^{*} \mathbb{P}^{1}$ is:

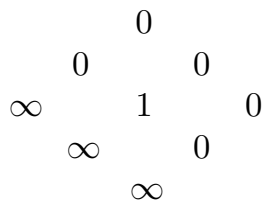


The KKP Hodge Diamond of the Landau-Ginzburg model on $\operatorname{LG}\left(\mathcal{O}_{2}\right)$ obtained from Thm. 4.3 was calculated in [BGRSM, Sec. 7] and is:

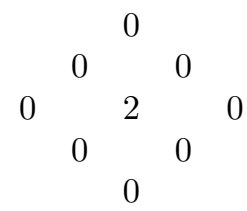

LG(2)

5.3.2. An example in 4 dimensions. Our LG model is $\left(\mathcal{O}_{3}, f_{H}\right)=$ : LG(3) obtained from Thm. 4.3 using the adjoint orbit $\mathcal{O}_{3}$ of $\operatorname{Diag}(2,-1,-1)$ with potential $f_{H}$ corresponding to the choice of $H=\operatorname{Diag}(1,0,-1)$.

The adjoint orbit $\mathcal{O}_{3}$ is a noncompact affine variety of dimension 4 that has the following Hodge diamond:

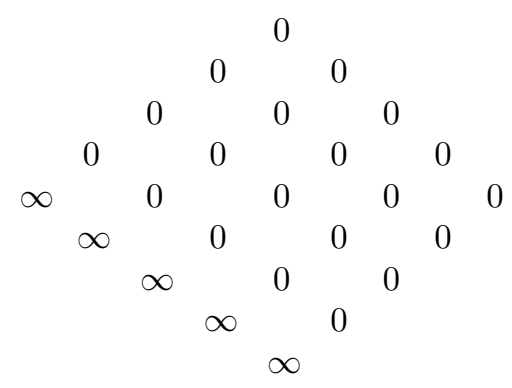

$\mathcal{O}_{3}$

The adjoint orbit $\mathcal{O}_{3}$ is a deformation of the cotangent bundle of $\mathbb{P}^{2}$, and $T^{*} \mathbb{P}^{2}$ has the following Hodge diamond:

$\begin{array}{cccccccccc} & & & & 0 & & & & \\ & & & 0 & & 0 & & & \\ & & 0 & & 0 & & 0 & & \\ & 0 & & 0 & & 0 & & 0 & \\ \infty & & 0 & & 1 & & 0 & & 0 \\ & \infty & & 0 & & 0 & & 0 & \\ & & \infty & & 1 & & 0 & & \\ & & & \infty & & 0 & & & \\ & & & & \infty & & & & \end{array}$

$\mathrm{T}^{*} \mathbb{P}^{2}$ 
LG(3) admits a tame compactification, and the corresponding KKP diamond calculated in [BGRSM, Sec. 7] is:

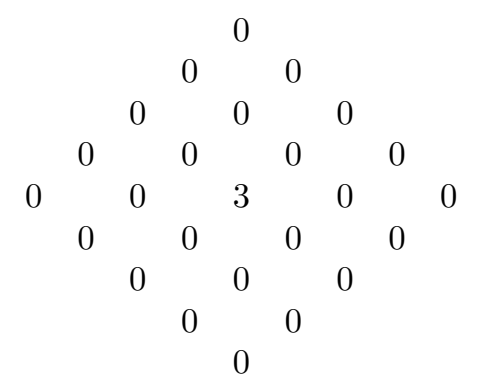

$\mathrm{LG}(3)$

5.3.3. The general case. [BGRSM] proved the KKP conjecture for minimal adjoint orbits of $\mathfrak{s l}(n, \mathbb{C})$. They calculated the KKP diamond of $\mathrm{LG}(n)$ to be:

0

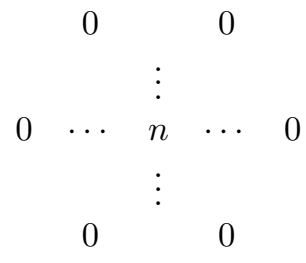

0

In conclusion, we have shown that our new concept of deformations of complex structures can be applied to many interesting examples. Furthermore, we have described classical Hodge theoretical invariants of cotangent bundles of projective spaces, and have compared them to the classical Hodge theoretical invariants of the nontrivial affine deformations of them, namely the minimal semisimple adjoint orbits of $\mathfrak{s l}(n, \mathbb{C})$. Finally we have described Landau-Ginzburg models on these adjoint orbits, and presented their KKP diamonds, that is, diamonds containing the three new invariants defined by $[\mathbf{K K P}]$, which as proved by [BGRSM], coincide for such orbits. How KKP diamonds vary under deformations is an interesting question that remains open. In fact, there are many delicate and intricate open questions about the deformation theory of Landau-Ginzburg models both in complex and in symplectic geometry.

\section{Acknowledgements}

We thank Severin Barmeier and the referee for suggesting several improvements to the text.

\section{References}

[BV] V. Balaji, P.A. Vishwanath, On the deformations of certain moduli spaces of vector bundles, Amer. J. Math. 115 (1993) 279-303.

[BBGGSM] E. Ballico, S. Barmeier, E. Gasparim, L. Grama, L. A. B. San Martin, A Lie theoretical construction of a Landau-Ginzburg model without projective mirrors, Manuscripta Math. 158 n.1-2 (2019) 85-101. 
[BCG] E. Ballico, B. Callander, E. Gasparim,Compactifications of adjoint orbits and their Hodge diamonds, J. Algebra Appl. 16 (2017) 1-17.

[BGK] E. Ballico, E. Gasparim, T. Köppe, Vector bundles near negative curves: moduli and local Euler characteristic, Comm. Algebra 37 (2009) 2688-2713.

[BG1] S. Barmeier, E. Gasparim, Classical deformations of local surfaces and their moduli of instantons, J. Pure Appl. Algebra 223 n.6 (2019) 2543-2561.

[BG2] S. Barmeier, E. Gasparim, Quantizations of local surfaces and rebel instantons, to appear in J. Noncommut. Geom. (2021).

[BGRSM] E. Ballico, E. Gasparim, F. Rubilar, L. A. B. San Martin, KKP conjecture for minimal adjoint orbits, arXiv:1901.07939.

[Bq] O. Biquard, Sur les équations de Nahm et la structure de Poisson des algèbres de Lie semisimples complexes, Math. Ann. 304 (1996) 253-276.

[Bo] F. A. Bogomolov, On the decomposition of Kähler manifolds with trivial canonical class, Mat. Sb. (N.S.), 93(135) n.4 (1974) 573-575.

[CP] I. Cheltsov, V. Przyjalkowski, Katzarkov-Kontsevich-Pantev Conjecture for Fano threefolds, arxiv:1809.09218.

[GGSM1] E. Gasparim, L. Grama, L. A. B. San Martin, Symplectic Lefschetz fibrations on adjoint orbits, Forum Math. 28 n.5 (2016) 967-980.

[GGSM2] E. Gasparim, L. Grama, L. A. B. San Martin, Adjoint orbits of semisimple Lie groups and Lagrangian submanifolds, Proc. Edinburgh Math. Soc. 60 (2) (2017) 361-385.

[GKRS] E. Gasparim, T. Köppe, F. Rubilar, B. Suzuki, Deformations of noncompact Calabi-Yau threefolds, Rev. Colombiana de Matemáticas 52 n.1 (2018) 41-57.

[GS] E. Gasparim, B. Suzuki, Infinite dimensionality of deformation families for noncompact threefolds, arXiv:1909.01842v2.

[H] J. E. Humphreys, Introduction to Lie algebras and representation theory, Springer-Verlag, New York, (1980).

[KKP] L. Katzarkov, M. Kontsevich, T. Pantev, Bogomolov-Tian-Todorov theorems for LandauGinzburg models, J. Differential Geom. 105 (1) (2017) 55-117.

[Ko] K. Kodaira, Complex manifolds and deformations of complex structures, Grundlehren der mathematischen Wissenschaften 283, Berlin: Springer (1986).

[Kv] A. G. Kovalev, Nahm's equations and complex adjoint orbits, Q. J. Math. 47 (1996) 41-58.

[Kr1] P. B. Kronheimer, A hyper-kählerian structure on adjoint orbits of a semisimple complex group, J. Lond. Math. Soc. (2) 42 (1990) 193-208.

[Kr2] P. B. Kronheimer, Instantons and the geometry of nilpotent variety, J. Differential Geom., 32 (1990) 473-490.

[LP] V. Lunts, V. Przyjalkowski, Landau-Ginzburg Hodge numbers for mirrors of del Pezzo surfaces, Adv. Math. 329 (2018) 189-216.

[M] M. Manetti, Lectures on deformations of complex manifolds (deformations from differential graded viewpoint), Rend. Mat. Appl. (7) 24 n.1 (2004) 1-183.

[NR] M.S. Narasimhan, S. Ramanan, Deformations of the moduli space of vector bundles over an algebraic curve, Ann. of Math. (2) 101 n.3 (1975) 391-417.

[R] F. Rubilar, Deformaciones de estructuras complejas de 3-variedades Calabi-Yau, Master Thesis, Universidad Católica del Norte, Chile (2017).

[SM] L. A. B. San Martin, Álgebras de Lie, Segunda edição, Editora Unicamp, ISBN 978-85-2680876-8 (2010), 454 pp.

Depto. Matemáticas, Univ. Católica del norte, Antofagasta, Chile.

Email address: etgasparim@gmail.com

Depto. Matemáticas, Univ. Católica del Norte, Antofagasta, Chile.

Email address: francisco.rubilar.arriagada@gmail.com 